\title{
Xilei San Ameliorates Experimental Colitis in Rats by Selectively Degrading Proinflammatory Mediators and Promoting Mucosal Repair
}

\author{
Yongbiao Hao, ${ }^{1,2}$ Kazuko Nagase, ${ }^{3}$ Kazutoshi Hori, ${ }^{3}$ Shenglan Wang, ${ }^{1,4}$ \\ Yoko Kogure, ${ }^{1,5}$ Ken Fukunaga, ${ }^{3}$ Shinichiro Kashiwamura, ${ }^{6}$ Satoshi Yamamoto, ${ }^{1}$ \\ Shiro Nakamura, ${ }^{3}$ Junxiang $\mathrm{Li}^{7}{ }^{7}$ Hiroto Miwa, ${ }^{2}$ Koichi Noguchi, ${ }^{4,5}$ and Yi Dai ${ }^{1,4,5}$ \\ ${ }^{1}$ Department of Pharmacy, School of Pharmacy, Hyogo University of Health Sciences, 1-3-6 Minatojima, Chuo-ku, Kobe, \\ Hyogo 650-8530, Japan \\ ${ }^{2}$ Division of Gastroenterology, Department of Internal Medicine, Hyogo College of Medicine, Nishinomiya, Hyogo 663-8501, Japan \\ ${ }^{3}$ Division of Internal Medicine, Department of Inflammatory Bowel Disease, Hyogo College of Medicine, Nishinomiya, \\ Hyogo 663-8501, Japan \\ ${ }^{4}$ Traditional Medicine Research Center, Chinese Medicine Confucius Institute at Hyogo College of Medicine, Kobe, \\ Hyogo 650-8530, Japan \\ ${ }^{5}$ Department of Anatomy and Neuroscience, Hyogo College of Medicine, Nishinomiya, Hyogo 663-8501, Japan \\ ${ }^{6}$ General Education Center, Hyogo University of Health Sciences, Kobe, Hyogo 650-8530, Japan \\ ${ }^{7}$ Department of Gastroenterology, Dongfang Hospital, Beijing University of Chinese Medicine, Beijing, 100078, China
}

Correspondence should be addressed to Yi Dai; ydai@huhs.ac.jp

Received 22 April 2014; Accepted 28 June 2014; Published 10 July 2014

Academic Editor: Yoshiji Ohta

Copyright (C) 2014 Yongbiao Hao et al. This is an open access article distributed under the Creative Commons Attribution License, which permits unrestricted use, distribution, and reproduction in any medium, provided the original work is properly cited.

\begin{abstract}
Xilei san (XLS), a herbal preparation widely used in China for erosive and ulcerative diseases, has been shown to be effective in ulcerative colitis (UC). The present experiments were conducted to assess its efficacy and determine its mechanism of action in a rat model that resembles human UC. The model was induced by adding $4 \%$ dextran sulfate sodium (DSS) to the rats' drinking water for 7 days. XLS was administered daily by retention enema from day 2 to day 7; the rats were sacrificed on day 8 . The colon tissues were obtained for further experiments. A histological damage score and the activity of tissue myeloperoxidase were used to evaluate the severity of the colitis. The colonic cytokine levels were detected in a suspension array, and epithelial proliferation was assessed using Ki-67 immunohistochemistry. Intrarectal administration of XLS attenuated the DSS-induced colitis, as evidenced by a reduction in both the histological damage score and myeloperoxidase activity. It also decreased the levels of proinflammatory cytokines, but increased the mucosal repair-related cytokines. In addition, the epithelial Ki-67 expression was upregulated by XLS. These results suggest that XLS attenuates DSS-induced colitis by degrading proinflammatory mediators and promoting mucosal repair. XLS could be a potential topical treatment for human UC.
\end{abstract}

\section{Introduction}

Inflammatory bowel disease (IBD) is characterized by chronic uncontrolled episodes of gastrointestinal inflammation [1]. Ulcerative colitis (UC), a major phenotype of IBD, is characterized by damage involving the mucosa and submucosa of the colon; bloody diarrhea is the major symptom. Inducing remission and preventing relapse are the primary goals in the management of UC. According to current consensus-based guidelines [2-4], the medical treatment for UC patients should take into consideration the clinical activity (mild, moderate, or severe) and the extent of colonic involvement (proctitis, left-sided colitis, or pancolitis). In patients with mild-to-moderate proctitis, mesalazine suppositories are often used as the first-line treatment. In a double-blind randomized study, we showed 
significant clinical and endoscopic efficacies of Xilei san (XLS) suppositories as well as their safety, in proctitis patients refractory to conventional topical therapy [5].

XLS is a compounded prescription of Chinese medicine, consisting of a fixed dose combination of the following well-characterized and standardized eight natural herbal or mineral substances: natural indigo (indigo naturalis), cow bezoar (calculus bovis), pearl powder (margarita), watermelon frost (mirabilitum praeparatum), calcitum (gypsum rubrum), borax (natrium biboricum), ammonium chloride (sal ammoniac), and borneol (borneolum syntheticum). In China, XLS is traditionally used in disorders that feature erosion or ulceration of mucosa or skin, including oral ulcer, esophagitis, erosive gastritis, peptic ulcer, chronic cervicitis, and UC [6-12]. Although a few studies have investigated the mechanism of action of XLS in an animal model [11], the exact pharmacological steps of XLS in UC have never been fully elucidated.

Clinical studies with herbal therapy in IBD have shown promising efficacy data and an acceptable safety profile; however, these remain limited and heterogeneous, and highquality animal trials are still lacking [13]. With this background in mind, we investigated the pharmacological mechanism of the anti-inflammatory effects and mucosal healing of XLS on rats with dextran sulfate sodium- (DSS-) induced acute colitis-a condition that shares not only clinical and structural features with human UC but also pathophysiological and immunological characteristics [14-16].

\section{Materials and Methods}

2.1. Experimental Animals. All the animal experimental procedures previously approved by the Committee on Animal Research in Hyogo University of Health Sciences (number 2010-22) were performed in accordance with the guidelines on animal care of the National Institutes of Health. Adult male Sprague-Dawley (SD) rats (200-250 g; Japan SLC, Inc., Shizuoka, Japan) were housed in collective cages (3 rats per cage) at $24 \pm 1^{\circ} \mathrm{C}$ under a $12 \mathrm{~h}$-light/dark cycle, with free access to food and sterile tap water. All animals were allowed to adapt for 3 days before the experiments were begun. All experiments were performed during the light phase of the cycle.

2.2. DSS-Induced Colitis. DSS (MW 36-50 KDa, MP Biomedical, California, USA) was dissolved in sterilized tap water and presented to the rats at a final concentration of $4 \%$ w/v for 7 consecutive days. Fresh DSS solution was provided every day. Negative control healthy rats (noncolitis) received only sterilized tap water.

2.3. Treatment Protocol. The rats were randomly allocated to 4 groups: control rats treated with saline (water + saline); control rats treated with XLS (water + XLS); rats with DSSinduced colitis treated with saline (DSS + saline); and rats with DSS-induced colitis treated with XLS (DSS + XLS). XLS was prepared as a powder from a product commercially supplied by Beijing Tong Ren Tang Group Co., Ltd., Beijing,
China. From day 1 to day 7, DSS solution was orally administered, ad libitum, to the DSS + saline and the DSS + XLS groups to induce colitis. Sterilized tap water was given to the rats in the other groups. On the following day, that is, day 2, the rats in the water + XLS and DSS + XLS groups were given XLS solution ( $0.3 \mathrm{~g}$ in $0.6 \mathrm{~mL}$ saline) intrarectally, while the remaining rats received only saline. After the rats were anesthetized intraperitoneally (i.p.) with pentobarbital sodium $(50 \mathrm{mg} / \mathrm{kg})$, the XLS solution was delivered to the colon at a depth of $8 \mathrm{~cm}$ from the anus, using a plastic circular needle. The retention enema, maintained for $2 \mathrm{~h}$, was given daily from day 2 to day 7 . After an overnight fast, the rats were killed on day 8 , as described below. Body weight and colon length were measured at day 8.

2.4. Histological Analysis for Scoring Inflammation. The rats were deeply anesthetized with pentobarbital sodium $(50 \mathrm{mg} / \mathrm{kg}$, i.p.) and then perfused transcardially with $250 \mathrm{~mL}$ of $0.1 \mathrm{M}$ phosphate buffer ( $\mathrm{pH}$ 7.4) containing $1 \%$ paraformaldehyde (Nacalai Tesque, Kyoto, Japan) followed by $500 \mathrm{~mL}$ of the same buffer containing $4 \%$ paraformaldehyde. Colon lengths of $8 \mathrm{~cm}$ were removed and cut into 6 segments, each measuring $0.5 \mathrm{~cm}$, starting from anus and cutting at $1 \mathrm{~cm}$ intervals. The sections were fixed in $4 \%$ buffered paraformaldehyde for 3 days at $4^{\circ} \mathrm{C}$. The tissues were then transferred to a $30 \%$ solution of sucrose $(\mathrm{w} / \mathrm{v})$ for 1 day for cryoprotection and then sliced on a cryostat at $6 \mu \mathrm{m}$ thickness. The sections were mounted on silanecoated slides and stored at $-80^{\circ} \mathrm{C}$ until used. After staining with hematoxylin and eosin (HE) (Wako Pure Chemical Industries, Ltd., Osaka, Japan), the degree of damage and inflammation was histologically assessed, and the histological damage score was calculated according to the following three parameters, which were scored on a scale of 0-4: (1) total lateral length of erosion, defined as loss of mucosal tissue $(0$, $0 \mathrm{~mm} ; 1,0.01-0.49 \mathrm{~mm}$; 2, 0.50-0.99 mm; 3, 1.00-1.49 mm; 4, $\geq 1.50 \mathrm{~mm}$ ); (2) total lateral length of inflammation, defined as a significantly excessive infiltration of leukocytes to the mucosa (0, $0 \mathrm{~mm} ; 1,0.01-0.99 \mathrm{~mm} ; 2,1.00-1.99 \mathrm{~mm} ; 3,2.00-$ $2.99 \mathrm{~mm} ; 4, \geq 3.00 \mathrm{~mm}$ ); and (3) a polymorphonuclear cell count of the densest mucosal area (high-power field, $\times 400$ ), $(0,0-29 ; 1,30-59 ; 2,60-89 ; 3,90-119 ; 4, \geq 120)$. The degree of histological damage was then scored from 0 to 12 .

2.5. Assay for Myeloperoxidase (MPO) Activity. Colonic MPO activity was assayed according to a method previously described [17]. Rats were sacrificed by decapitation. A $4 \mathrm{~cm}$ length of colon, measured from the anus, was removed and washed with phosphate-buffered saline over ice. The colonic mucosa was scraped off with a glass slide and then suspended in $1 \mathrm{~mL}$ of $50 \mathrm{mM}$ potassium phosphate buffer ( $\mathrm{pH} 6.0$ ) containing $0.5 \%$ hexadecyl trimethyl ammonium bromide (Sigma Chemical Co., St. Louis, Missouri, USA). The suspension was next homogenized with an ultrasonic homogenizer and then centrifuged. MPO activity in the supernatant was assayed spectrophotometrically at $25^{\circ} \mathrm{C}$ by mixing $0.1 \mathrm{~mL}$ of the supernatant with $2.9 \mathrm{~mL}$ of $50 \mathrm{mM}$ phosphate buffer $(\mathrm{pH}$ 6.0) containing $0.167 \mathrm{mg} / \mathrm{mL}$-dianisidine dihydrochloride 
(Sigma Chemical Co., St. Louis, Missouri, USA) and 0.0005\% hydrogen peroxide. The change in absorbance at $460 \mathrm{~nm}$ was measured after $15 \mathrm{~min}$ with a spectrophotometer (Beckman Coulter, Inc., California, USA). The results are expressed as optical density (OD) per total protein of tissue.

2.6. Measurement of Cytokines and Chemokines. Rats were anesthetized with pentobarbital $(50 \mathrm{mg} / \mathrm{kg})$ and killed by decapitation. The $2 \mathrm{~cm}$ length colon was removed starting from anus, washed with wash buffer (Bio-Rad Laboratories, Inc., Hercules, California, USA), and homogenized in Bio-Plex cell lysis buffer (Bio-Rad Laboratories, Inc., Hercules, California, USA). After centrifugation, the supernatants were collected and stored at $-80^{\circ} \mathrm{C}$ until further use. The supernatants were used to assay cytokines and chemokines using Bio-Plex Pro Rat Cytokine 24Plex Assay kit (Bio-Rad Laboratories, Inc., Hercules, California, USA). The following cytokines and chemokines were quantified: interleukin- (IL-) $1 \alpha$, IL-1 $\beta$, IL-2, IL-4, IL-5, IL-6, IL-7, IL-10, IL-12, IL-13, IL-17, IL-18, erythropoietin (EPO), granulocyte-colony stimulating factor (GCSF), granulocyte macrophage-colony stimulating factor (GM-CSF), growth-related oncogene/keratinocyte-derived chemokine $(\mathrm{GRO} / \mathrm{KC})$, interferon- $\gamma(\mathrm{IFN}-\gamma)$, macrophage colony-stimulating factor (M-CSF), monocyte inflammatory protein-1 $\alpha$ (MIP-1 $\alpha$ ), MIP-3 $\alpha$, regulated on activation normal $\mathrm{T}$ cell expressed and secreted (RANTES), tumor necrosis factor (TNF)- $\alpha$, vascular endothelial growth factor (VEGF), and monocyte chemoattractant protein (MCP-1).

2.7. Ki-67 Immunohistochemistry. Frozen sections $(6 \mu \mathrm{m})$ of colon were made, as described above, and processed for $\mathrm{Ki}$ 67 immunohistochemistry. The procedure was described in detail in a previous study [18]. A 1:500 solution of the primary monoclonal antibody of Ki-67 (clone MM1; Novocastra Laboratories, Newcastle, UK) was used. After reacting with diaminobenzidine, the sections were counterstained with hematoxylin, washed in Milli-Q water (Millipore Corporation, Billerica, MA, USA), air-dried, and dehydrated via an alcohol gradient $(70,80,90,95$, and $100 \%)$. After the alcohol was replaced by xylene, the sections were coverslipped. The labeling index (LI) was calculated according to a previously described method [19] and expressed as a percentage of the positive cells among 1000 cells. To remove an observation bias, epithelial cells on tubules, which had a U-shaped configuration to enable a fair observation from the surface to the basal side, were counted close to the erosive or inflamed area.

2.8. Statistical Analysis. All data are reported as mean \pm standard error (SE). The Steel-Dwass test was used for nonparametric all-pairs multiple comparison in each group of data. Differences of $P<0.05$ or less were considered to be significant. The statistical tests were performed using JMP Software version 10.0 (SAS Institute Inc., Cary, North Carolina, USA).

\section{Results}

3.1. Body Weight and Colon Length. Body weight was significantly loosed in the DSS group compared with the control group (DSS group versus water + saline group: $245.8 \pm 10.5 \mathrm{~g}$ $[N=11]$ versus $268.5 \pm 17.7 \mathrm{~g}[N=10], P<0.05)$. Likewise, in the DSS group, colon length $(\mathrm{cm})$ was significantly shorter compared with the water + saline group (DSS group versus water + saline group: $16.8 \pm 1.2 \mathrm{~cm}[N=11]$ versus $19.2 \pm$ $1.6 \mathrm{~cm}[N=10], P<0.05)$. However, quantification of the body weight or the colon length did not demonstrate significant alteration between DSS group and DSS + XLS group (body weight: DSS group versus DSS + XLS group: $245.8 \pm 10.5 \mathrm{~g}[N=11]$ versus $231.2 \pm 21.8 \mathrm{~g}[N=12]$, $P=0.13$, colon length: DSS group versus DSS + XLS group: $16.8 \pm 1.2 \mathrm{~cm}[N=11]$ versus $17.0 \pm 0.8 \mathrm{~cm}[N=12]$, $P=0.90)$.

3.2. Histological Features. Representative histological images of HE-stained colon sections from each group are shown in Figure 1. In contrast to that of the control group (Figure 1(a)), the histology of tissues from the DSS-induced colitis revealed erosions and a remarkable infiltration of inflammatory cells with some increase of fibroblasts (Figure 1(c)). In the DSS + XLS group, after intrarectal administration of XLS, crypt regeneration and restoration with mild infiltration of inflammatory cells were observed (Figure $1(\mathrm{~d})$ ). Interestingly, the water + XLS group showed a significant accumulation of Peyer's patches in the submucosa (Figure 1(b)). As shown in the bar graph (Figure 1(e)), the histological damage score increased significantly more in the DSS + saline group than in the water + saline group $(9.80 \pm 0.70[N=10]$ versus $1.43 \pm 0.48[N=7], P<0.01)$. Treatment with XLS tended to protect against the histological damage induced by DSS, when compared to the DSS + saline group $(7.33 \pm 0.82[N=9]$ versus $9.80 \pm 0.70[N=10], P=0.18$, not statistically significant).

3.3. Decrease in Colonic MPO Activity in DSS-Treated Rats due to XLS. As expected, the MPO activity in colonic mucosa was significantly greater in the DSS + saline group than in the water + saline group $(0.21 \pm 0.02 \mathrm{OD} /$ total protein $[N=11]$ versus $0.05 \pm 0.01[N=10], P<0.01)$. This difference was significantly less when the group given DSS + XLS was compared to the one given DSS + saline $(0.06 \pm 0.01$ $\mathrm{OD} /$ total protein $[N=12]$ versus $0.21 \pm 0.02[N=11]$, $P<0.01$ ) (Figure 2). These results suggest that XLS inhibits granulocytic infiltration in DSS-induced colitis.

3.4. XLS Suppression of the Increase in Inflammatory Cytokines and Chemokines Induced by DSS. Proinflammatory cytokine IL- $1 \beta$, which is secreted mainly by macrophages, may contribute to the tissue damage seen in DSS-induced colitis. The concentration of IL- $1 \beta$ in colonic tissue was significantly greater in the DSS + saline group than that in the water + saline group $(4528.05 \pm 1089.04 \mathrm{pg} / \mathrm{mg}$ protein $[N=9]$ versus $1413.61 \pm 256.04[N=10], P<0.01)$. A comparison of the IL- $1 \beta$ induced by DSS with and without XLS treatment 


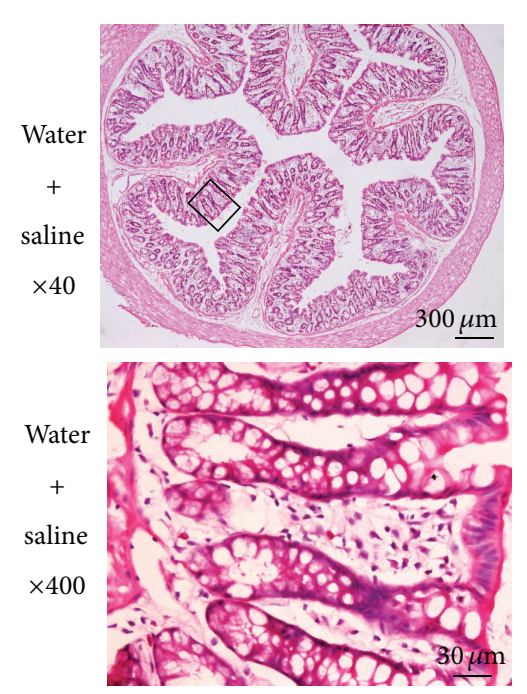

(a)
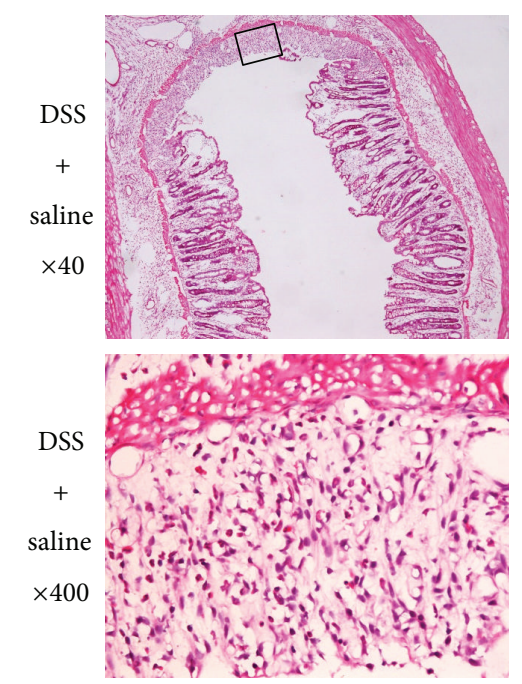

(c)

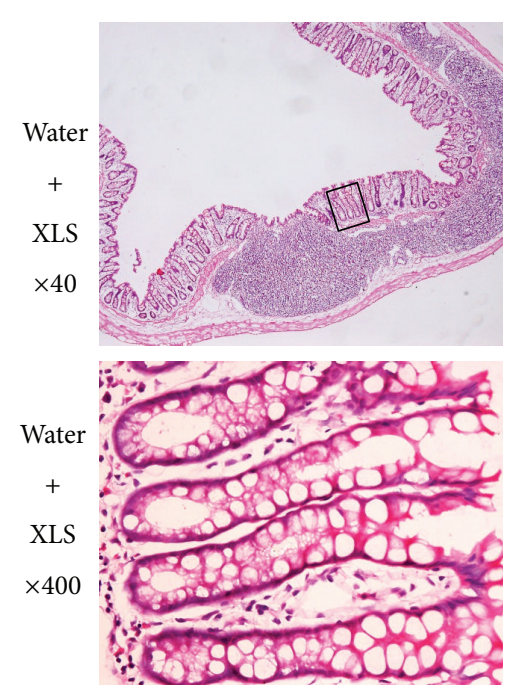

(b)
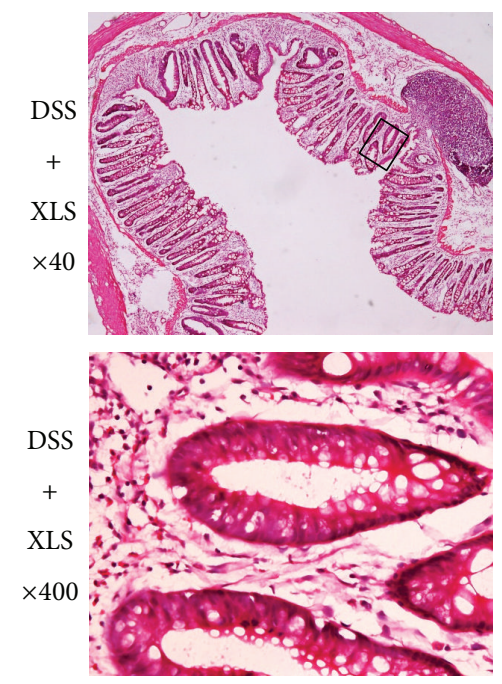

(d)

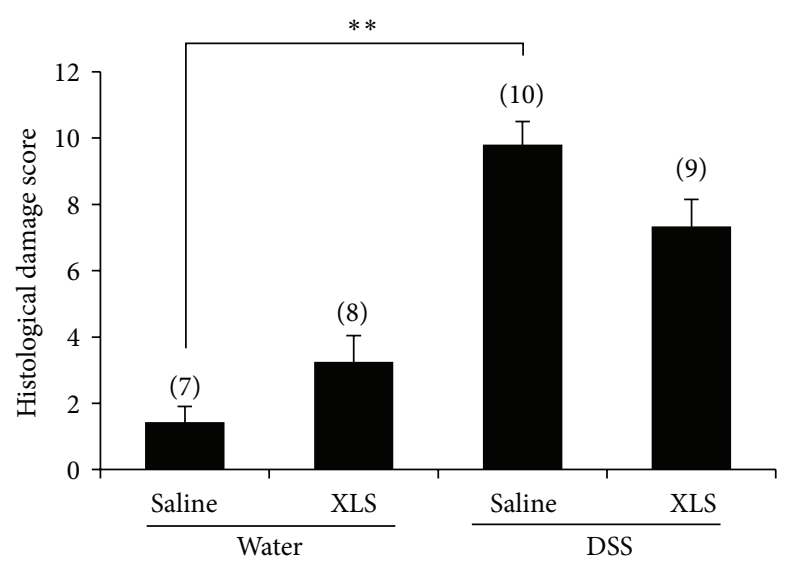

(e)

FIGURE 1: XLS attenuated DSS-induced colonic mucosal damage in rats. (a) Water + saline (sterile tap water and saline); (b) water + XLS (sterile tap water and $0.3 \mathrm{~g}$ XLS); (c) DSS + saline (4\% DSS and saline); (d) DSS + XLS (4\% DSS and $0.3 \mathrm{~g}$ XLS) (HE staining, $\times 40$ and $\times 400$ original magnification); and (e) total histological damage score. Numbers in brackets represent the sample numbers of each group. ${ }^{* *} P<0.01$ versus water + saline. 


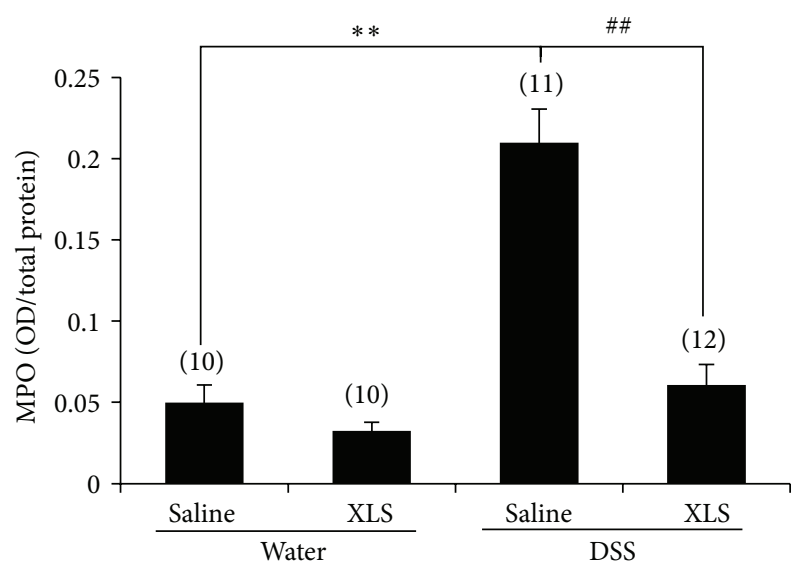

FIGURE 2: XLS reduced colonic MPO activity in DSS-treated rats. Numbers in brackets represent the sample numbers of each group. ${ }^{* *} P<0.01$ versus water + saline, ${ }^{\# \#} P<0.01$ versus DSS + saline.

shows that XLS prevents the elevation of IL- $1 \beta$, as evidenced by the values for IL-1 $\beta$ in the DSS + XLS and the DSS + saline groups $(1571.09 \pm 70.72 \mathrm{pg} / \mathrm{mg}$ protein $[N=10]$ versus $4528.05 \pm 1089.04[N=9], P<0.01$ ) (Figure 3(a)). The concentration of other proinflammatory cytokines, such as IL-6, IL-17, and TNF- $\alpha$, was not significantly elevated in the DSS + saline group compared with water + saline group $(847.28 \pm 118.13 \mathrm{pg} / \mathrm{mg}$ protein $[N=9]$ versus $654.19 \pm 74.44$ $[N=10], P=0.64$ for IL- $6 ; 115.37 \pm 10.93 \mathrm{pg} / \mathrm{mg}$ protein $[N=9]$ versus $149.73 \pm 11.87[N=10], P=0.34$ for IL-17, and $1888.52 \pm 162.45 \mathrm{pg} / \mathrm{mg}$ protein $[N=9]$ versus $2313.05 \pm 171.16[N=10], P=0.43$ for TNF- $\alpha$, resp.) (Figures 3(b)-3(d)).

In addition to the differences in the concentration of a proinflammatory cytokine IL- $1 \beta$, two colonic chemokinesGRO/KC (Figure 4(a)) and MCP-1 (Figure 4(b)) - were significantly greater in the DSS + saline group than in the water + saline group $(817.92 \pm 308.07 \mathrm{pg} / \mathrm{mg}$ protein $[N=9]$ versus $84.99 \pm 4.73[N=10], P<0.01$ for $\mathrm{GRO} / \mathrm{KC}$, and $2065.03 \pm 316.12 \mathrm{pg} / \mathrm{mg}$ protein $[N=9]$ versus $391.63 \pm 59.02$ $[N=10], P<0.01$ for MCP-1, resp.). Both GRO/KC and $\mathrm{MCP}-1$ protein levels were suppressed by XLS, as seen when comparing the two groups, DSS + XLS, and DSS + saline $(155.31 \pm 15.38 \mathrm{pg} / \mathrm{mg}$ protein $[N=10]$ versus $817.92 \pm 308.07[N=9], P<0.01$ for $\mathrm{GRO} / \mathrm{KC}$, and $666.11 \pm$ $45.73 \mathrm{pg} / \mathrm{mg}$ protein $[N=10]$ versus $2065.03 \pm 316.12[N=$ 9], $P<0.01$ for MCP-1, resp.).

3.5. XLS Upregulated Colonic Mucosal Repair-Related Cytokine Expression and Spurred Enterocyte Proliferation in DSS-Treated Rats. VEGF is a cytokine that promotes angiogenesis and MIP-3 $\alpha$ functions in epithelial migration and mucosal barrier repair. Treatment with DSS reduced colonic VEGF (Figure 5(a)) and MIP-3 $\alpha$ (Figure 5(b)) levels, as seen in the comparison between the assays for these moieties in the DSS + saline and water + saline groups $(57.95 \pm 14.20 \mathrm{pg} / \mathrm{mg}$ protein $[N=9]$ versus $147.42 \pm 12.66$ $[N=10], P<0.01$ for VEGF, and $511.64 \pm 124.53 \mathrm{pg} / \mathrm{mg}$ protein $[N=9]$ versus $1781.36 \pm 364.39[N=10]$,
$P<0.05$ for MIP- $3 \alpha$ resp.), while XLS treatment significantly prevented that degradation, as evidenced by comparing the values obtained from the DSS + XLS and DSS + saline groups $(117.75 \pm 12.71 \mathrm{pg} / \mathrm{mg}$ protein $[N=10]$ versus $57.95 \pm 14.20$ $[N=9], P<0.05$ for VEGF, and $1358.20 \pm 147.28 \mathrm{pg} / \mathrm{mg}$ protein $[N=10]$ versus $511.64 \pm 124.53[N=9], P<0.05$ for MIP-3 $\alpha$, resp.). Furthermore, XLS treatment upregulated the MIP- $3 \alpha$ expression in normal rats, as seen in the comparison of the MIP- $3 \alpha$ from the water + XLS and the water + saline groups $(3668.15 \pm 265.42 \mathrm{pg} / \mathrm{mg}$ protein $[N=9]$ versus $1781.36 \pm 364.39[N=10], P<0.01)$ (Figure 5(b)).

$\mathrm{Ki}-67$, a nuclear protein expressed in all active phases of the cell cycle, is necessary for cell proliferation. To explore whether or not XLS could interfere with enterocyte proliferation, we evaluated the Ki-67 expression in colonic epithelium as a function of the LI. Ki-67 immunohistochemistry exhibited low expression in epithelial cells in the water + saline group (Figure 6(a)). In contrast, DSS treatment increased the Ki-67 expression as seen by comparing the water + saline and the DSS + saline groups (Figure 6(c)) $(27 \pm 2 \%$ in LI $[N=7]$ versus $43 \pm 3 \%[N=10], P<0.01$ in Figure 6(e)). Interestingly, XLS, as well, promoted the expression of Ki-67, as evidenced by the comparison between the water + saline and water + XLS groups (Figure 6(b)) $(27 \pm 2 \%$ in LI [ $N=7]$ versus $40 \pm 3 \%[N=8], P<0.01$ in Figure 6(e)). The expression in the DSS + XLS group was even greater than that in the DSS + saline group (Figure $6(\mathrm{~d}))(43 \pm 3 \%$ in LI $[N=10]$ versus $50 \pm 2 \%[N=9], P=0.19)$, although the difference was not statistically significant (Figure 6(e)). These data indicate that XLS stimulates enterocyte proliferation in DSS-induced colitis (Figure 6(d)).

\section{Discussion}

In the present study, we demonstrated that intrarectal administration of XLS suppresses DSS-induced colitis by decreasing the infiltration and migration of inflammatory cells into the colon, reducing colonic MPO activity, degrading the proinflammatory cytokines and chemokines, and promoting mucosal repair.

4.1. XLS Reduction of Local Infiltration, Migration of Inflammatory Cells, and Production of Inflammatory Cytokines. Inflammation of the intestinal mucosa is characterized by an excessive infiltration of such inflammatory cells as neutrophils and macrophages, which is accompanied by the production of proinflammatory cytokines [20]. Neutrophils play a crucial role in mediating tissue injury and clinical symptoms in colitis [21-23]. In the histological analysis, we found that neutrophilic infiltration was increased in the DSS rat colonic tissue. XLS treatment reduced this infiltration (Figure 1(d)). Neutrophilic infiltration correlates with tissue MPO activity, which represents the severity of DSS-induced colitis [24]. Although MPO is an enzyme that catalyzes the formation of hypochlorous acid, it also possesses cytokinelike properties and can activate neutrophils, with a resulting release of a wide range of inflammatory mediators [25]. In 


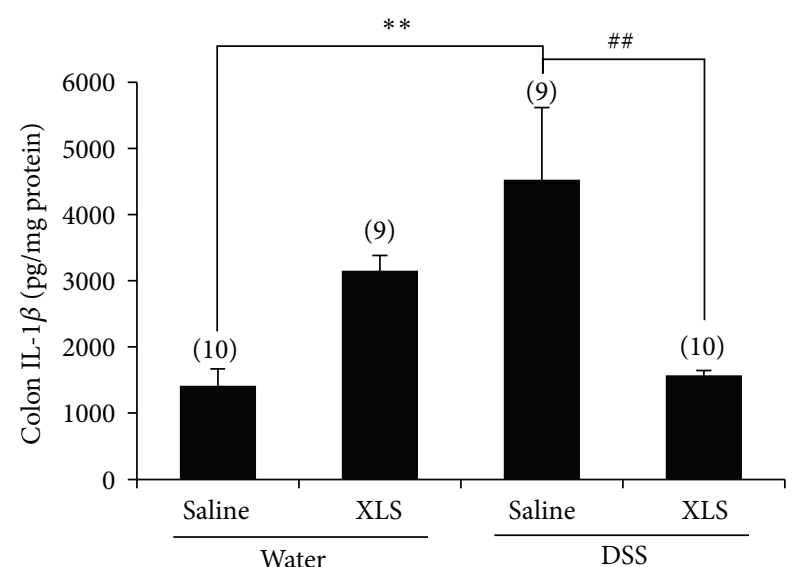

(a)

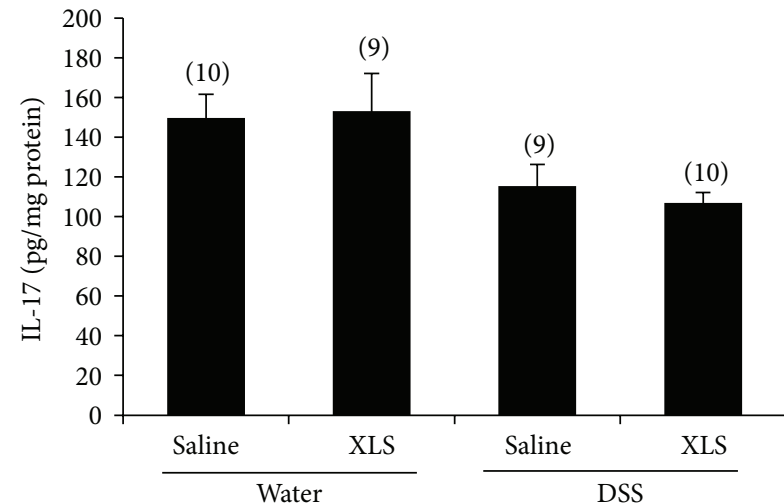

(c)

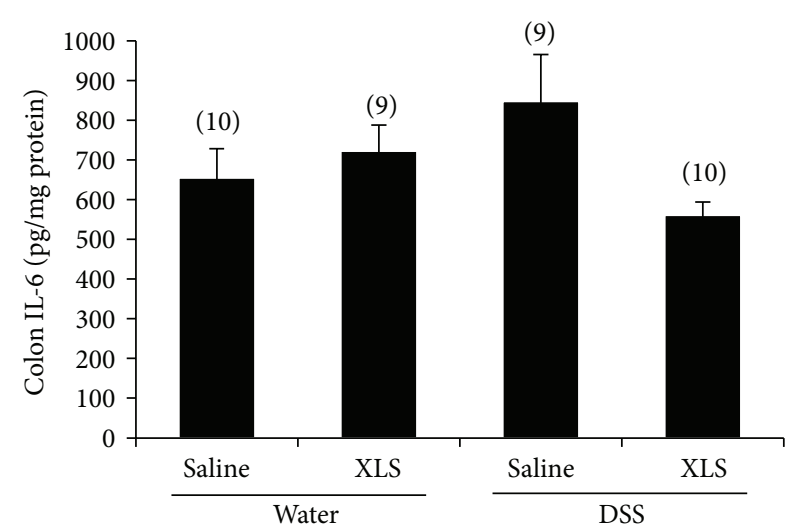

(b)

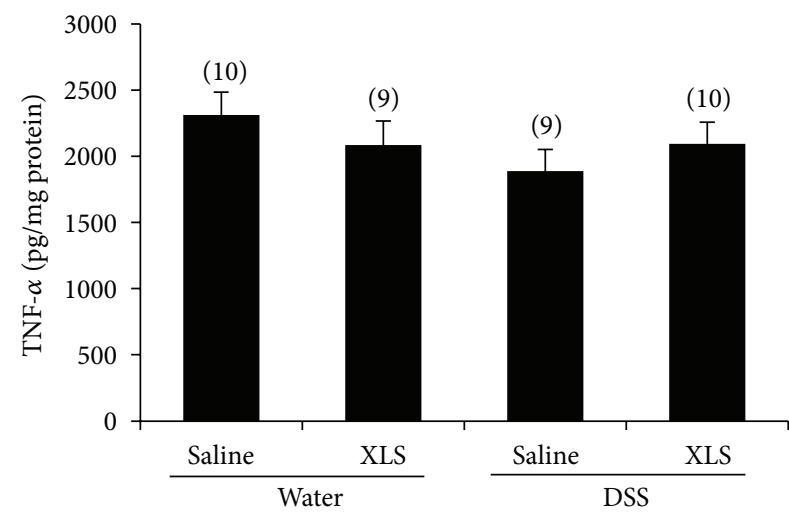

(d)

FIGURE 3: In rats, XLS suppressed the increase of a colonic cytokine of IL-1 $\beta$ (a) induced by DSS. IL-6 (b), IL-17 (c), and TNF- $\alpha$ (d) remained constant in DSS group compared with water group, which was comparable to that of XLS groups. Numbers in brackets represent the sample numbers of each group. ${ }^{* *} P<0.01$ versus water + saline, ${ }^{\# \#} P<0.01$ versus DSS + saline.

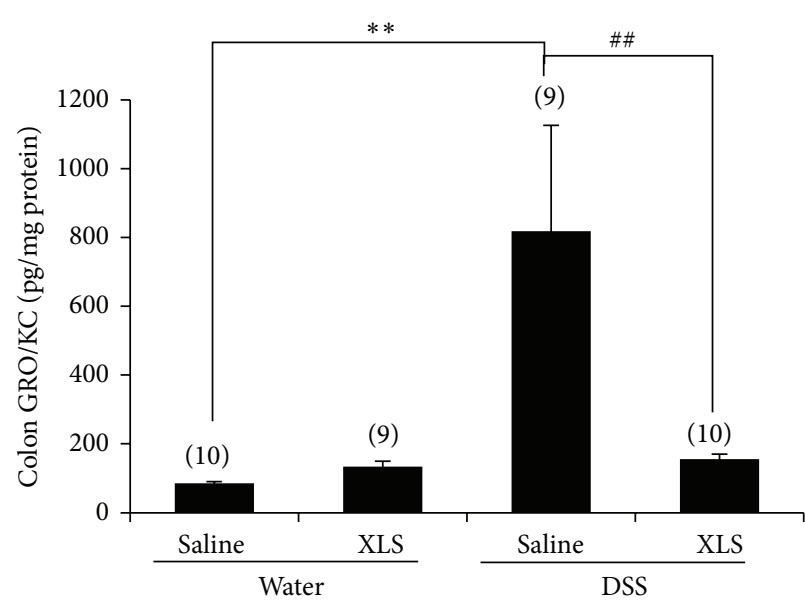

(a)

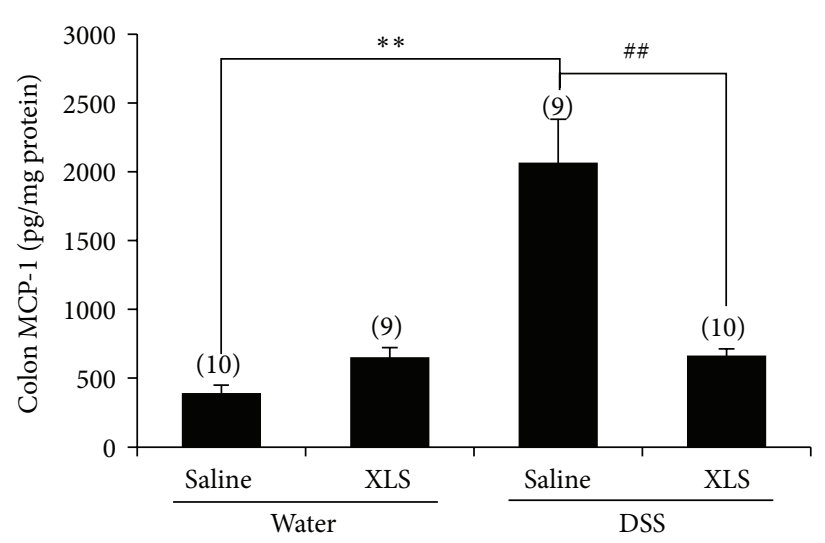

(b)

FIGURE 4: In rats, XLS inhibited the increase of the colonic chemokines (a) GRO/KC and (b) MCP-1 induced by DSS. Numbers in brackets represent the sample numbers of each group. ${ }^{* *} P<0.01$ versus water + saline, ${ }^{\# \#} P<0.01$ versus DSS + Saline. 


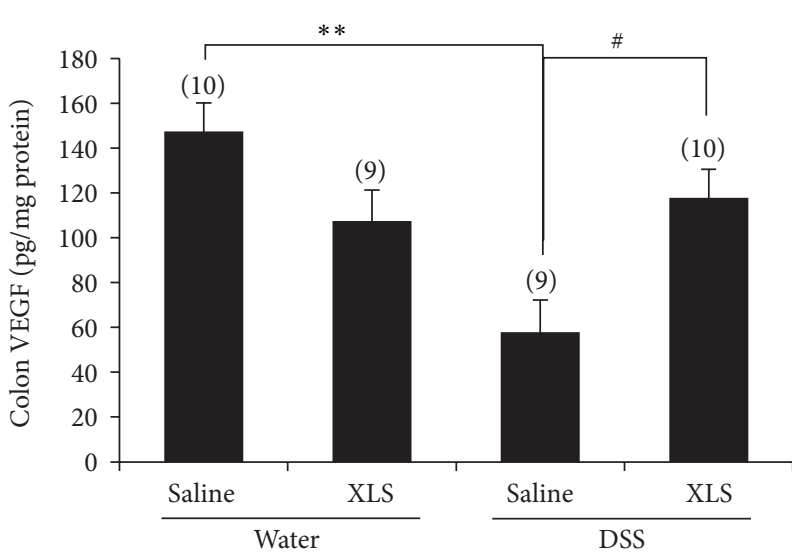

(a)

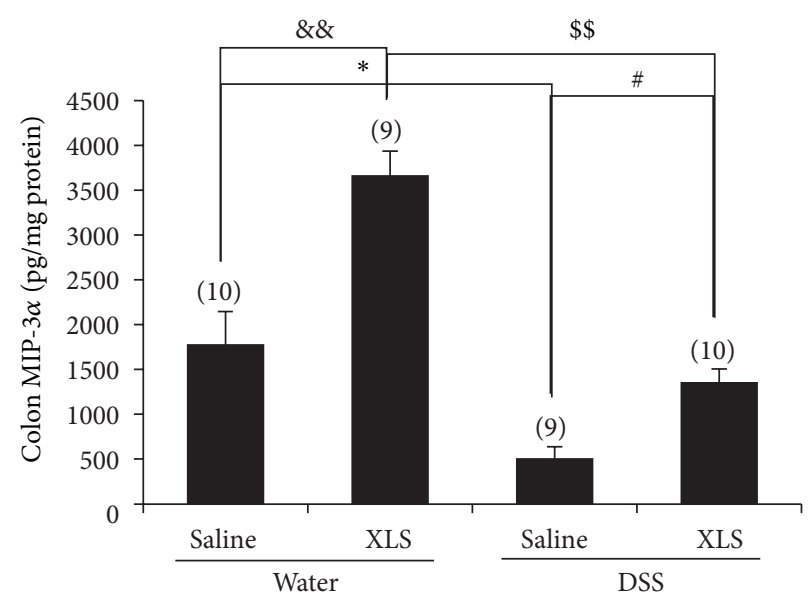

(b)

FIGURE 5: In rats, XLS upregulated colonic mucosal repair-related cytokine expression of (a) VEGF and (b) MIP-3 $\alpha$. Numbers in brackets represent sample numbers of each group. ${ }^{* *} P<0.01$ versus water + saline, ${ }^{*} P<0.05$ versus water + saline, ${ }^{\#} P<0.05$ versus DSS + saline, ${ }^{\& \&} P<0.01$ versus water + saline, and ${ }^{\$ \$} P<0.01$ versus DSS + XLS.

accord with this evidence, MPO activity was shown to significantly increase in rat colonic tissue with DSS-induced colitis, and the XLS treatment prevented this increase (Figure 2), a finding indicative of a reduction in neutrophilic infiltration and a decrease in colon damage.

Activated inflammatory cells may upregulate the production of cytokines in colon tissue, and cytokines then create a positive feedback loop that exacerbates the colonic damage [26]. Circulating IL-1 $\beta$, IL-6, IL-17, and TNF- $\alpha$ play a key role in the pathogenesis of IBD [27]. Since IL-1 $\beta$ is involved in the early stage of DSS-induced colitis, the downregulation might be available for the treatment of patients with UC [28]. We found that XLS treatment significantly reduced IL- $1 \beta$ production in DSS-induced colitis, a finding that could indicate that XLS prevents neutrophil and macrophage infiltration. It is reported that IL-6 levels correlate with IBD activity [29]. Although upward tendency of IL-6 level was found in the DSS group, there was no significant difference compared with water group (Figure 3(b)). This may indicate a mild severity of inflammation in the present DSS model. On the other hand, IL-17 is a delayed-type immune reaction cytokine produced by Th17 and by CD8+ T cells during chronic inflammation $[29,30]$. DSS colitis represents an acute model of colitis [30]; this idea supports that we could not detect significant changes of IL-17 expression on the present DSS colitis. In the present study, we evaluate the proinflammatory cytokines at 7 days after DSS treatment, the time point which may represent the early stage of the colitis. DSS may activate local and infiltrated macrophage to produce IL-1 $\beta$ prior to upregulation of other proinflammatory cytokines in this time point. Regarding these points of view, the colitis model in the present study may represent the early stage and/or mild severity of inflammation. Further studies are warranted to investigate the effect of XLS on these proinflammatory cytokines, such as IL-6, IL-17, and TNF- $\alpha$ in colitis.

The inflammatory cells in DSS-induced colitis may be chemically attracted to the intestinal mucosa by chemokines.
In the present study, we observed that GRO/KC and MCP-1 were upregulated by DSS, while XLS significantly prevented the production of both GRO/KC and MCP-1 in DSS-induced colitis (Figure 4). Given that $\mathrm{GRO} / \mathrm{KC}$ is a chemokine for neutrophils [31], while MCP-1 plays a chemoattractant role for macrophages [32], these findings suggest that XLS can reduce the infiltration of neutrophils and macrophages to the inflamed foci. Therefore, the XLS treatment may contribute to a decrease in cell migration by diminishing the production of chemotactic factors.

\subsection{XLS Promotion of Epithelial Repair in the Damaged} Colon. Intestinal mucosa has a zone of rapidly proliferating epithelial cells, which sustains injuries in response to stresses ranging from physiological daily digestive trauma to severe insults associated with ischemia, chemical insult, and infection. Mucosal repair in the acute phase of colitis is a complex process and often includes villus contraction, epithelial migration, and closure of the epithelial cell gap and tight junctions [33]. These processes require cytokines such as VEGF and MIP- $3 \alpha$. VEGF plays a pivotal role in the reconstruction of vascular cells [34], while MIP-3 $\alpha$ contributes to efficient epithelial migration and mucosal barrier repair [35]. In the present study, we found that the expressions of VEGF and MIP- $3 \alpha$ were markedly downregulated in DSSinduced colitis, while XLS treatment significantly inhibited the downregulation of these cytokines (Figure 5). These results indicate that XLS promotes epithelial repair. In addition to this observation, XLS treatment notably increased $\mathrm{Ki}$ 67 protein expression in colonic epithelium, with or without DSS-induced colitis. Ki-67, a nuclear protein associated with cellular proliferation, is present during all active phases of the cell cycle (G1, S, G2, and mitosis) but is absent from resting cells (G0) [36]. Therefore, the pathway underlying the XLS protection against colonic damage could be associated with proliferating enterocytes. Taken as a whole, these data 


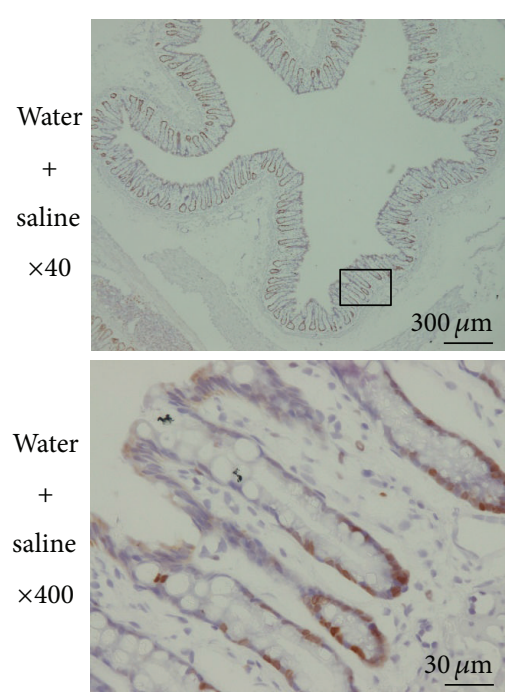

(a)

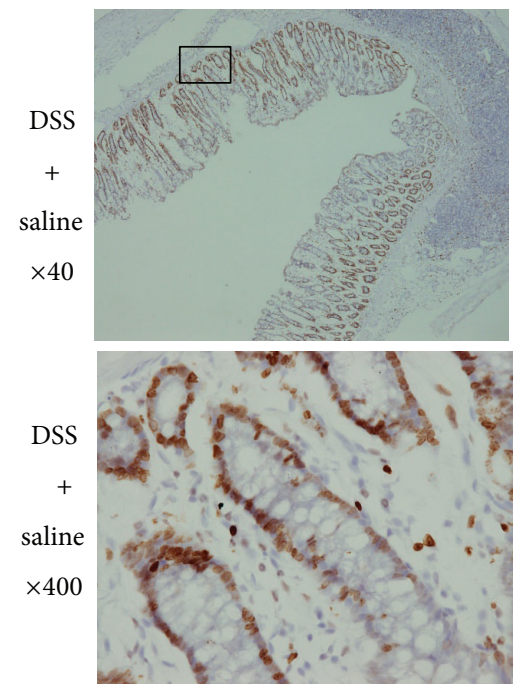

(c)

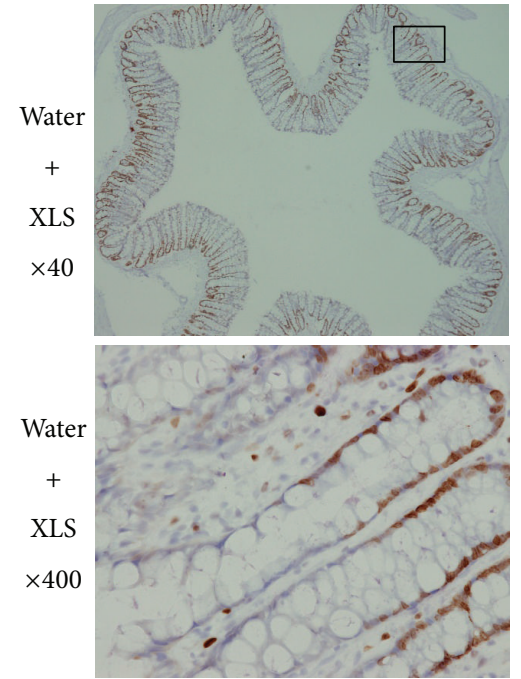

(b)

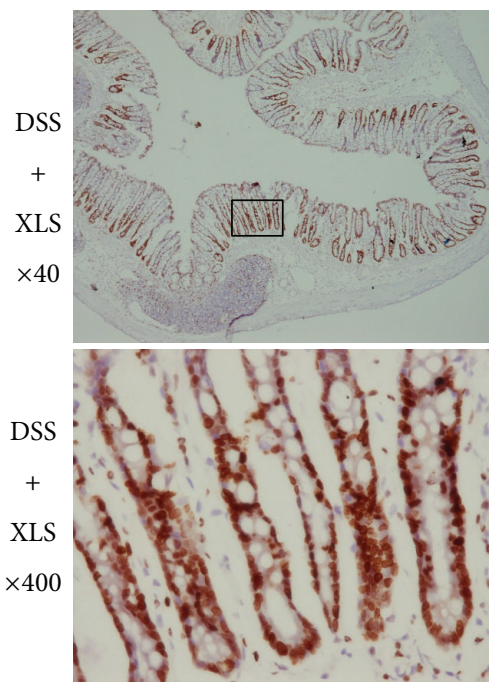

(d)

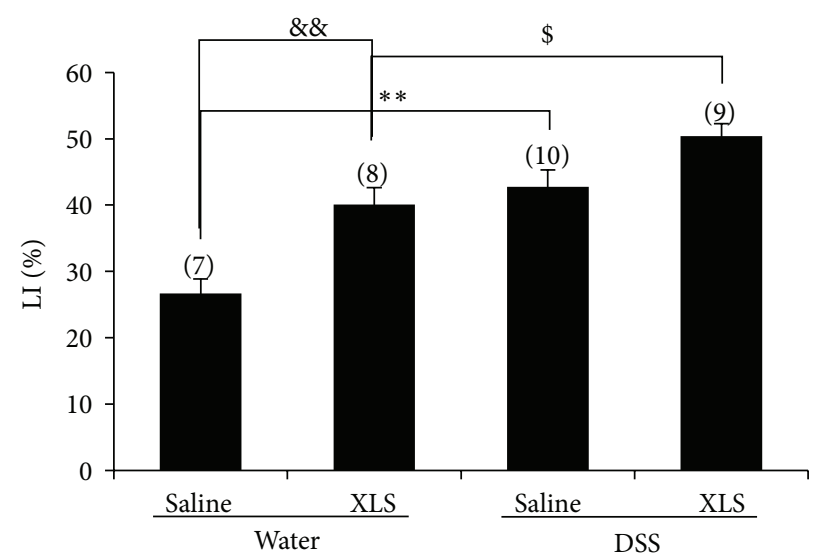

(e)

FIGURE 6: XLS stimulated enterocyte proliferation in DSS-treated rats. (a) Water + saline; (b) water + XLS; (c) DSS + saline; and (d) DSS + XLS (immunohistochemistry, $\times 40$ and $\times 400$ original magnification). (e) Cell proliferation was evaluated by LI, representative of the immunoreactivity of Ki-67 in colon sections. Numbers in brackets represent sample numbers of each group. ${ }^{* *} P<0.01$ versus water + saline, ${ }^{\& \&} P<0.01$ versus water + saline, and ${ }^{\$} P<0.05$ versus DSS + XLS. 
indicate that XLS would be effective in promoting epithelial migration and mucosal barrier repair.

It was noteworthy that XLS treatment increased the expression of colonic MIP-3 $\alpha$ (Figure 5(b)). In connection with the finding, we observed that lymphocytes accumulated in the submucosa (also known as Peyer's patch) in the XLStreated colon (Figure $1(\mathrm{~b})$ ). MIP- $3 \alpha$ is strongly chemotactic for lymphocytes and thus implicated in the formation and function of lymphoid tissue via the chemoattraction of lymphocytes and dendritic cells toward the epithelial cells around lymphoid tissue [37]. This accumulation of lymphocytes may participate in mucosal restoration by releasing mucosal repair-related cytokines, but determining the exact mechanism will require further investigation.

In this study, we assessed only the efficacy of the action of XLS on DSS-induced colitis in the acute phase [15]. Therefore, whether XLS exerts its action in the chronic phase by the same mechanism is still unclear. In addition, although we confirmed the efficacy of topical XLS administration on DSSinduced colitis, we need to investigate further whether or not systemic administration of the compound would also be effective. Moreover, because XLS is a mixture of Chinese medical herbs that include eight natural herbal or mineral substances, we cannot know without further investigation whether any one component or one substance in one component plays a special role in the healing process.

\section{Conclusions}

Our results show that topical treatment with XLS can ameliorate colitis. The action is associated with two possible mechanisms. First, XLS could prevent the release in colonic tissue of proinflammatory mediators, by inhibiting the influx of leukocytes into inflamed foci. Second, XLS may promote both mucosal repair and epithelial cell proliferation, which contribute to the mucosal restoration. Considering the evidence from our previous clinical reports $[5,38]$, we suggest XLS as a candidate for the topical treatment of UC.

\section{Conflict of Interests}

The authors declare that there is no conflict of interests regarding the publication of this paper.

\section{Acknowledgments}

This work was supported by the Research Grant from Chinese Medicine Confucius Institute at Hyogo College of Medicine, the Heiwa Nakajima Foundation and the Science Research Grant for work on intractable diseases, and the Japanese Ministry of Health, Labour and Welfare. Yongbiao Hao was supported by the SENSHU Scholarship Foundation and the research assistant program of Hyogo College of Medicine. Part of these data have been presented at the 134th annual meeting of the pharmaceutical society of Japan as a poster presentation.

\section{References}

[1] W. Strober, I. Fuss, and P. Mannon, "The fundamental basis of inflammatory bowel disease," Journal of Clinical Investigation, vol. 117, no. 3, pp. 514-521, 2007.

[2] A. Kornbluth and D. B. Sachar, "Gastroenterology PPCotACo. Ulcerative colitis practice guidelines in adults: American College of Gastroenterology, Practice Parameters Committee," The American Journal of Gastroenterology, vol. 105, no. 3, pp. 501$524,2010$.

[3] C. Mowat, A. Cole, A. Windsor et al., "Guidelines for the management of inflammatory bowel disease in adults," Gut, vol. 60 , no. 5, pp. 571-607, 2011.

[4] S. P. Travis, E. F. Stange, M. Lemann et al., "European evidencebased Consensus on the management of ulcerative colitis: current management," Journal of Crohn's and Colitis, vol. 2, no. 1, pp. 24-62, 2008.

[5] K. Fukunaga, Y. Ohda, N. Hida et al., "Placebo controlled evaluation of Xilei San, a herbal preparation in patients with intractable ulcerative proctitis," Journal of Gastroenterology Hepatology, vol. 27, no. 12, pp. 1808-1815, 2012.

[6] Y. E. Zhen, "Xileisan and zinc gluconate used in treating relapsing oral ulcer," Anhui Medical and Pharmaceutical Journal, vol. 2005, no. 5, p. 9, 2005.

[7] L. S. Zhang and E. S. Bao, "Status quo of traditional Chinese treatment for radioactive esophagitis," Shanghai Journal of Traditional Chinese Medicine, no. 5, pp. 24-25, 2007.

[8] D. M. Li and S. Q. Li, "Clinical analysis of Xileisan matching smectite powder in treatment for the patients with chronic erosive gastritis," Medical Recapitulate, vol. 2011, no. 4, p. 52, 2011.

[9] A. T. Zhang, L. X. Qian, and J. M. Guan, "Study on therapeutic mechanism of Xi Lei powder for peptic ulcer," Chinese Journal of Integrated Traditional and Western Medicine, vol. 15, no. 4, pp. 216-218, 1995.

[10] Y. P. Gao, H. F. Ni, L. Y. Zhu Mao, and Z. X. Jiang, "Explore the drug effect mechanism of Xilei San treating chronic cervicitis," Chinese Archives of Traditional Chinese Medicine, vol. 2007, no. 8, p. 37, 2007.

[11] Y. Pan and Q. Ouyang, "Effects of Bawei Xilei San on mice with oxazolone-induced colitis and the mechanisms," Journal of Chinese Integrative Medicine, vol. 8, no. 6, pp. 568-574, 2010.

[12] F. Zhang, Y. Li, F. Xu, Y. Chu, and W. Zhao, "Comparison of Xilei-san, a Chinese herbal medicine, and dexamethasone in mild/moderate ulcerative proctitis: a double-blind randomized clinical trial," The Journal of Alternative and Complementary Medicine, vol. 19, no. 10, pp. 838-842, 2013.

[13] S. C. Ng, Y. T. Lam, K. K. Tsoi et al., "Systematic review: the efficacy of herbal therapy in inflammatory bowel disease," Alimentary Pharmacology and Therapeutics, vol. 38, no. 8, pp. 854-863, 2013.

[14] C. O. Elson, R. B. Sartor, G. S. Tennyson, and R. H. Riddell, "Experimental models of inflammatory bowel disease," Gastroenterology, vol. 109, no. 4, pp. 1344-1367, 1995.

[15] E. Gaudio, G. Taddei, A. Vetuschi et al., "Dextran sulfate sodium (DSS) colitis in rats: clinical, structural, and ultrastructural aspects," Digestive Diseases and Sciences, vol. 44, no. 7, pp. 1458$1475,1999$.

[16] T. Ohkusa, "Production of experimental ulcerative colitis in hamsters by dextran sulfate sodium and changes in intestinal microflora," The Japanese Journal of Gastro-Enterology, vol. 82, no. 5, pp. 1327-1336, 1985. 
[17] P. P. Bradley, D. A. Priebat, R. D. Christensen, and G. Rothstein, "Measurement of cutaneous inflammation: estimation of neutrophil content with an enzyme marker," Journal of Investigative Dermatology, vol. 78, no. 3, pp. 206-209, 1982.

[18] Y. Ohda, K. Hori, T. Tomita et al., "Effects of hepatocyte growth factor on rat inflammatory bowel disease models," Digestive Diseases and Sciences, vol. 50, no. 5, pp. 914-921, 2005.

[19] H. Goishi, S. Tanaka, K. Haruma et al., "Predictive value of cathepsin D and Ki-67 expression at the deepest penetration site for lymph node metastases in gastric cancer," Oncology Reports, vol. 7, no. 4, pp. 713-718, 2000.

[20] M. A. McGuckin, R. Eri, L. A. Simms, T. H. Florin, and G. Radford-Smith, "Intestinal barrier dysfunction in inflammatory bowel diseases," Inflammatory Bowel Diseases, vol. 15, no. 1, pp. 100-113, 2009.

[21] J. E. Krawisz, P. Sharon, and W. F. Stenson, "Quantitative assay for acute intestinal inflammation based on myeloperoxidase activity. Assessment of inflammation in rat and hamster models," Gastroenterology, vol. 87, no. 6, pp. 1344-1350, 1984.

[22] J. L. Wallace, W. McKnight, S. Asfaha, and D. Y. Liu, "Reduction of acute and reactivated colitis in rats by an inhibitor of neutrophil activation," American Journal of Physiology, vol. 274, no. 5, part 1, pp. G802-G808, 1998.

[23] B. M. Fournier and C. A. Parkos, "The role of neutrophils during intestinal inflammation," Mucosal Immunology, vol. 5, no. 4, pp. 354-366, 2012.

[24] I. Villegas, C. Alarcon de la Lastra, A. Orjales, and C. La Casa, "A new flavonoid derivative, dosmalfate, attenuates the development of dextran sulphate sodium-induced colitis in mice," International Immunopharmacology, vol. 3, no. 13-14, pp. 1731-1741, 2003.

[25] D. Lau, H. Mollnau, JP. Eiserich et al., "Myeloperoxidase mediates neutrophil activation by association with CD11b/CD18 integrins," Proceedings of the National Academy of Sciences of the United States of America, vol. 102, no. 2, pp. 431-436, 2005.

[26] S. Danese and C. Fiocchi, "Ulcerative colitis," The New England Journal of Medicine, vol. 365, no. 18, pp. 1713-1725, 2011.

[27] W. Shen and S. K. Durum, "Synergy of IL-23 and Th17 cytokines: new light on inflammatory bowel disease," Neurochemical Research, vol. 35, no. 6, pp. 940-946, 2010.

[28] Y. Arai, H. Takanashi, H. Kitagawa, and I. Okayasu, "Involvement of interleukin-1 in the development of ulcerative colitis induced by dextran sulfate sodium in mice," Cytokine, vol. 10, no. 11, pp. 890-896, 1998.

[29] L. de Fazio, E. Cavazza, E. Spisni et al., "Longitudinal analysis of inflammation and microbiota dynamics in a model of mild chronic dextran sulfate sodium-induced colitis in mice," World Journal of Gastroenterology, vol. 20, no. 8, pp. 2051-2061, 2014.

[30] M. Coccia, O. J. Harrison, C. Schiering et al., "IL-1 $\beta$ mediates chronic intestinal inflammation by promoting the accumulation of IL-17A secreting innate lymphoid cells and CD4(+) Th17 cells," The Journal of Experimental Medicine, vol. 209, no. 9, pp. 1595-1609, 2012.

[31] K. De Filippo, A. Dudeck, M. Hasenberg et al., "Mast cell and macrophage chemokines CXCL1/CXCL2 control the early stage of neutrophil recruitment during tissue inflammation," Blood, vol. 121, no. 24, pp. 4930-4937, 2013.

[32] M. Hori, H. Nobe, K. Horiguchi, and H. Ozaki, "MCP1 targeting inhibits muscularis macrophage recruitment and intestinal smooth muscle dysfunction in colonic inflammation," The American Journal of Physiology-Cell Physiology, vol. 294, no. 2, pp. C391-C401, 2008.
[33] N. Ferrara, "VEGF and the quest for tumour angiogenesis factors," Nature Reviews Cancer, vol. 2, no. 10, pp. 795-803, 2002.

[34] A. J. Moeser, M. M. Haskell, D. E. Shifflett et al., "ClC-2 chloride secretion mediates prostaglandin-induced recovery of barrier function in ischemia-injured porcine ileum," Gastroenterology, vol. 127, no. 3, pp. 802-815, 2004.

[35] R. A. Vongsa, N. P. Zimmerman, and M. B. Dwinell, "CCR6 regulation of the actin cytoskeleton orchestrates human beta defensin-2- and CCL20-mediated restitution of colonic epithelial cells," The Journal of Biological Chemistry, vol. 284, no. 15, pp. 10034-10045, 2009.

[36] B. P. Willing and A. G. Van Kessel, "Enterocyte proliferation and apoptosis in the caudal small intestine is influenced by the composition of colonizing commensal bacteria in the neonatal gnotobiotic pig," Journal of Animal Science, vol. 85, no. 12, pp. 3256-3266, 2007.

[37] M. Baba, T. Imai, M. Nishimura et al., "Identification of CCR6, the specific receptor for a novel lymphocyte-directed CC chemokine LARC," The Journal of Biological Chemistry, vol. 272, no. 23, pp. 14893-14898, 1997.

[38] K. Fukunaga, N. Hida, K. Ohnishi et al., "A suppository Chinese medicine (xilei-san) for refractory ulcerative proctitis: a pilot clinical trial," Digestion, vol. 75, no. 2-3, pp. 146-147, 2007. 


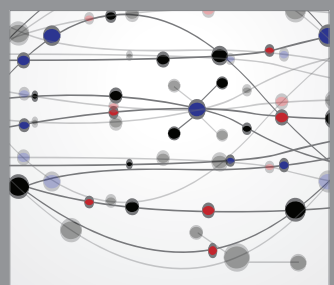

The Scientific World Journal
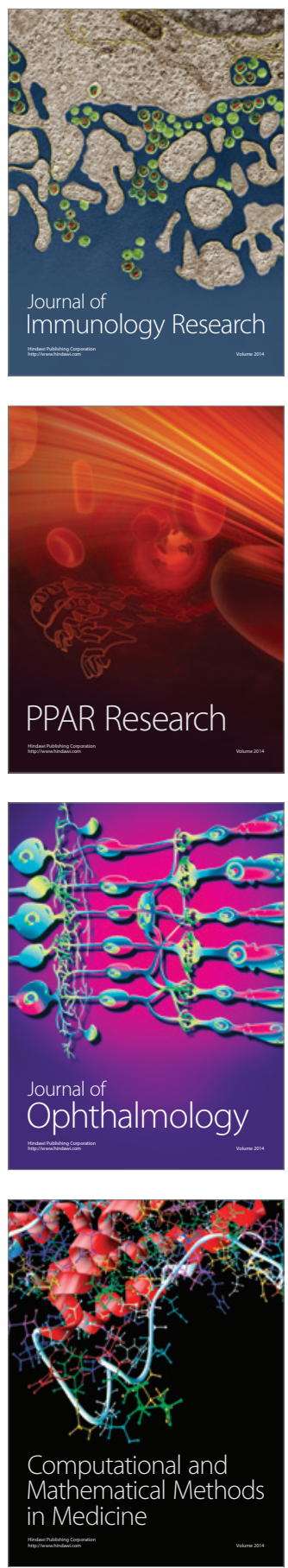

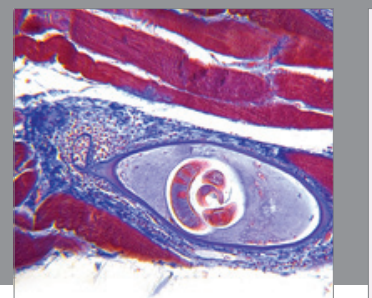

Gastroenterology

Research and Practice
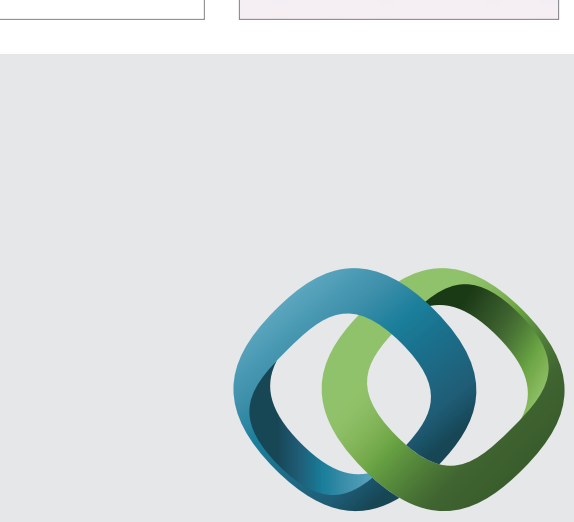

\section{Hindawi}

Submit your manuscripts at

http://www.hindawi.com
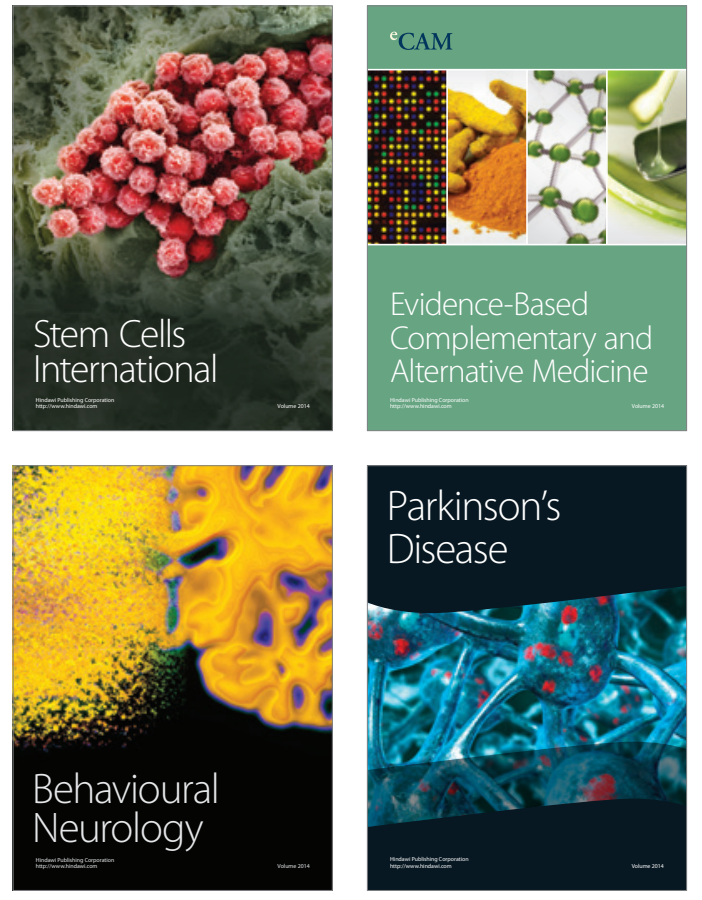
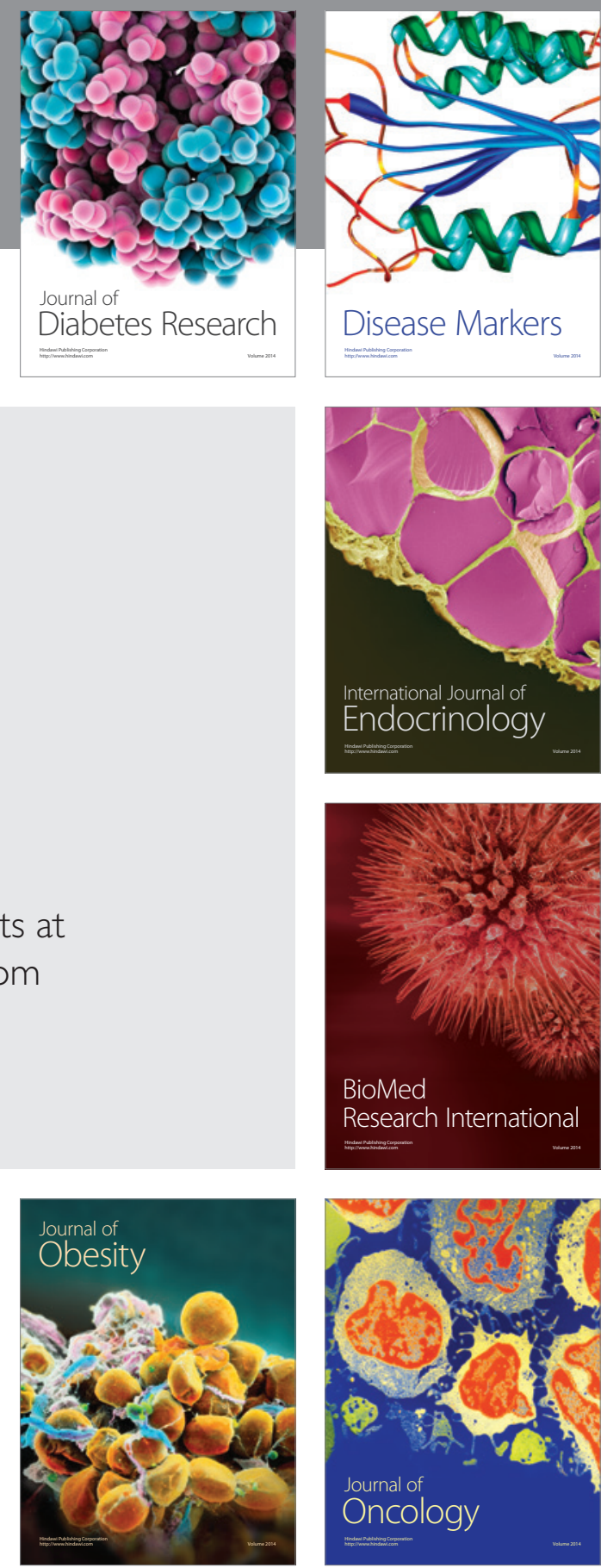

Disease Markers
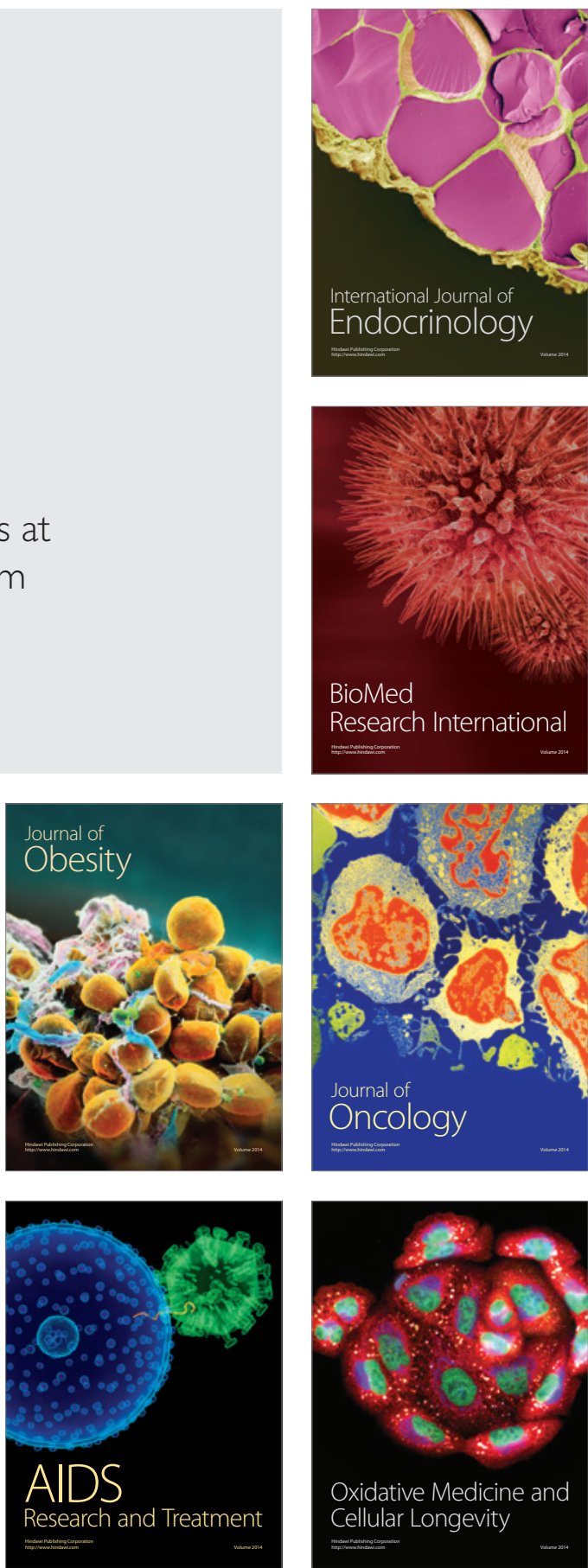